\title{
CT REGISTRATION: EXPERIMENTAL DETERMINATION OF SUITED FIDUCIAL MARKER MATERIAL AND REGISTRATION ERRORS
}

\author{
Maarten M. Arnolli*, Michel Franken* and Dannis M. Brouwer*i
}

\section{BACKGROUND}

CT imaging is commonly used by physicians to monitor the development of pathological conditions, e.g. brain tumours. Accurate monitoring of a unique point in the brain over consecutive CT scans in between which a patient's head may have moved requires determination of the relative position and orientation of the skull coordinate system with respect to the imager coordinate system, known as registration. A configuration of fiducial markers can be attached to the skull to provide means for registration by fitting of its known geometry with image data obtained from CT scanning. Typical requirements on fiducial based registration systems are related to fiducial and target registration errors and the extent to which the surrounding image is unaffected.

In [1], six $0.80 \mathrm{~mm}$ tantalum spherical fiducials were placed in phantom bones to register data from CT and roentgen stereogrammetric analysis, achieving a root mean square fiducial registration error of $0.152 \mathrm{~mm}$. Some reconstruction artefacts in the CT images are reported due to the high absorption rate of tantalum. In [2] and [3], registration is performed based on a single image using a configuration of obliquely placed aluminium rods. In [2], an average 3D position accuracy better than $1.2 \mathrm{~mm}$ is obtained with $50 \%$ of outliers in the data. An average displacement error at the instrument tip of $0.630 \mathrm{~mm}$ over 63 trials was reported in [3] with $95 \%$ of the errors under $1.0 \mathrm{~mm}$. Both registration systems require all rods to intersect with the image plane, limiting the range of rotation of the connected instrument for which registration is possible.

\footnotetext{
*DEMCON Advanced Mechatronics, Zutphenstraat 25, 7575 EJ, Oldenzaal, The Netherlands. Address all correspondence to Maarten.Arnolli@demcon.nl.

${ }^{\dagger}$ Mechanical Automation \& Mechatronics, Faculty of Engineering Technology, University of Twente, Horstring Z230, 7500 AE, Enschede, The Netherlands.
}

We propose the use of a configuration of three or more spherical fiducials to provide means for registration in a CT image set. This paper presents an experiment designed to determine which material is suited for use as a fiducial and what fiducial and target registration errors can be achieved given a particular configuration of fiducials.

\section{METHODS}

Firstly, fiducials of different material are CT imaged to choose a suitable material. Secondly, a set of CT images of a frame holding the fiducials of the chosen material is obtained in which the centre coordinates of the fiducials are localized by image processing. The frame coordinate system is registered using the known relative configuration of the fiducials as measured by a coordinate measuring machine. Fiducial registration errors are calculated directly in the registration process. Target registration errors are calculated by successively excluding one of the fiducials in the registration process and appointing it as a target.

\section{RESULTS}

A polyamide frame to hold $10 \mathrm{~mm}$ fiducials was produced by selective laser sintering. Fig. 1(a) shows the frame with fiducials being CT scanned by a Siemens Sensation 16 at the Universitair Medisch Centrum Groningen (UMCG) in the Netherlands (slice spacing $1.5 \mathrm{~mm}$, pixel spacing $0.95 \times 0.95 \mathrm{~mm}$, slice thickness 2 $\mathrm{mm}$ ). Fig. 2 shows cropped CT images of fiducials of nine different materials. Polymer fiducials yield relatively low pixel values which complicates their localization. Dark bands due to beam hardening appear predominantly between fiducials of zirconium dioxide and tungsten carbide in the same image plane. With high pixel values and no visible beam hardening, glass, silicon nitride and aluminium oxide are suitable materials. 


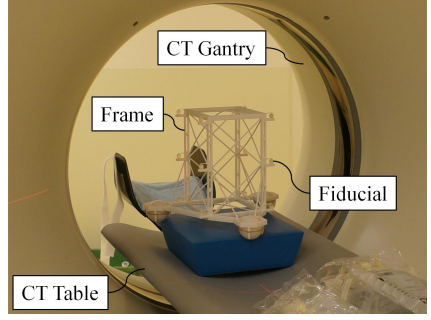

(a) Initial laser sintered PA frame.

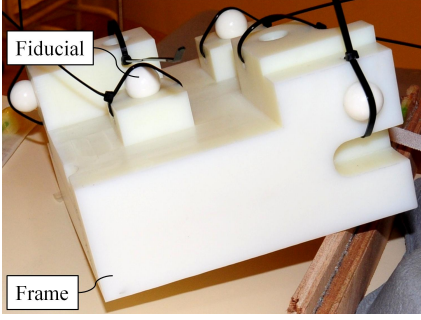

(b) Second machined POM frame.
FIGURE 1. INITIAL AND SECOND FRAME AND FIDUCIAL MARKERS PLACED IN A CT SCANNER AT THE UMCG.

The initial frame proved to be structurally too compliant and dimensionally unstable to obtain repeatable measurement results of the centre coordinates of the fiducials. A second more solid frame, shown in Fig. 1(b), was machined of polyoxymethylene and contains four $20 \mathrm{~mm}$ aluminium oxide fiducials at a centre to centre distance less than $230 \mathrm{~mm}$. The centre coordinates of each fiducial $x_{i}^{f}$ with respect to the frame coordinate system were obtained using a FARO GagePlus measuring arm with a specified volumetric length measuring uncertainty of $5 \mu \mathrm{m}$.

The frame with fiducials was CT imaged using the same CT and image settings as previously, yielding an image set in which the centre coordinates of the fiducials $\boldsymbol{x}_{i}^{c}$ with respect to the image coordinate system were determined by cropping of the $3 \mathrm{D}$ image set around each fiducial and averaging over the position of all pixels with values above a threshold value of 2476 Hounsfield units. The 3D position $\boldsymbol{x}_{f}^{c}$ and orientation in terms of Euler parameters $\lambda_{f}^{c}$ of the frame with respect to the CT coordinate system were determined using the iterative NewtonRaphson method to minimize a cost function $J$, which sums the squared values of the fiducial registration error $\boldsymbol{e}_{i}$ of $n$ fiducials:

$$
\begin{gathered}
\boldsymbol{e}_{i}\left(\boldsymbol{x}_{f}^{c}, \boldsymbol{\lambda}_{f}^{c}\right)=\boldsymbol{x}_{i}^{c}-\left(\boldsymbol{x}_{f}^{c}+\boldsymbol{R}^{f 2 c}\left(\boldsymbol{\lambda}_{f}^{c}\right) \cdot \boldsymbol{x}_{i}^{f}\right), \\
J\left(\boldsymbol{x}_{f}^{c}, \boldsymbol{\lambda}_{f}^{c}\right)=\sum_{i=1}^{n}\left\|\boldsymbol{e}_{i}\left(\boldsymbol{x}_{f}^{c}, \boldsymbol{\lambda}_{f}^{c}\right)\right\|^{2}, \\
\nabla J\left(\boldsymbol{x}_{f}^{c}, \boldsymbol{\lambda}_{f}^{c}\right)=0 .
\end{gathered}
$$

Here, $\boldsymbol{R}^{f 2 c}\left(\boldsymbol{\lambda}_{f}^{c}\right)$ is a rotation matrix which performs the rotation of coordinates from the frame coordinate system to the CT coordinate system, as a function of Euler parameters.

Four registrations have been performed using three out of four fiducials and the fourth appointed as a target. The fiducial and target registration errors are presented in Table 1, with an average of $0.10 \mathrm{~mm}$ and $0.20 \mathrm{~mm}$ respectively.

\section{INTERPRETATION}

The presented registration system yields no negative effects on CT images and achieves submillimetric fiducial and target registration errors. This accuracy is deemed sufficient for clinical procedures involving tracking points of interest over consecutive CT scans.

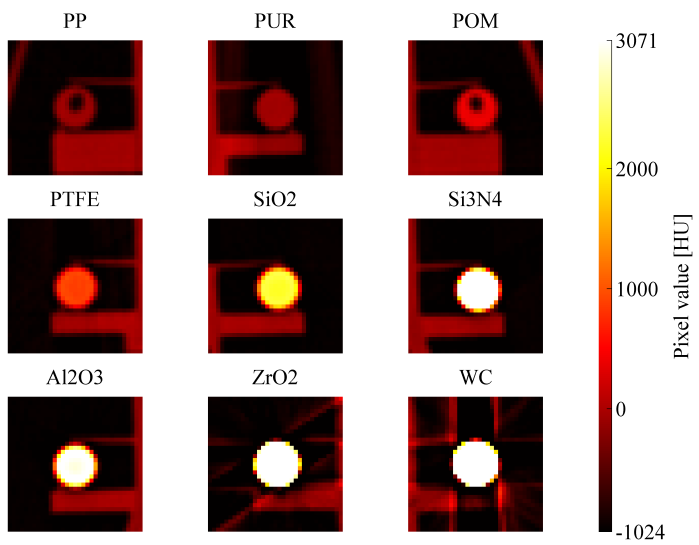

FIGURE 2. CROPPED CT IMAGES OF 10 MM FIDUCIAL MARKERS OF NINE DIFFERENT MATERIALS.

TABLE 1. SET $S$ OF FIDUCIALS USED FOR REGISTRATION VERSUS FIDUCIAL REGISTRATION ERRORS AND TARGET REGISTRATION ERRORS (IN BOLD) [MM].

\begin{tabular}{ccccc}
\hline$S$ & $\left\|\boldsymbol{e}_{1}\right\|$ & $\left\|\boldsymbol{e}_{2}\right\|$ & $\left\|\boldsymbol{e}_{3}\right\|$ & $\left\|\boldsymbol{e}_{4}\right\|$ \\
\hline$\{2,3,4\}$ & $\mathbf{0 . 1 3}$ & 0.14 & 0.10 & 0.12 \\
$\{1,3,4\}$ & 0.06 & $\mathbf{0 . 2 4}$ & 0.07 & 0.09 \\
$\{1,2,4\}$ & 0.13 & 0.11 & $\mathbf{0 . 2 2}$ & 0.09 \\
$\{1,2,3\}$ & 0.10 & 0.15 & 0.05 & $\mathbf{0 . 2 1}$ \\
\hline
\end{tabular}

\section{ACKNOWLEDGEMENTS}

The authors thank Martijn Buijze and Joost Könemann at DEMCON Advanced Mechatronics and Koert de Jong and Wim Tukker at the UMCG for their help in design and execution of the presented experiment.

\section{REFERENCES}

[1] R. Ellis, S. Toksvig-Larsen, M. Marcacci, D. Caramella, and M. Fadda. Use of a biocompatible fiducial marker in evaluating the accuracy of $\mathrm{CT}$ image registration. Investigative Radiology, 31(10):658-667, 1996.

[2] B. Maurin, C. Doignon, M. de Mathelin, and A. Gangi. A fast and automatic stereotactic registration with a single CT-slice. Computer Vision and Image Understanding, 113(8):878-890, 2009.

[3] R. Susil, J. Anderson, and R. Taylor. A single image registration method for CT guided interventions. In Proceedings of the International Conference on Medical Image Computing and Computer-Assisted Intervention, pages 798-808, 1999. 